Note d'information

\title{
MISE EN PLACE D'UNE DÉMARCHE QUALITÉ DANS LE CADRE DE L'ÉCHANTILLONNAGE D'UNE POPULATION DE GRANDE ALOSE (ALOSA ALOSA).
}

\author{
C. GAZEAU (1), T. LEFRANÇOIS (1, 2), E. ROCHARD (1)
}

(1) Cemagref, Unité Ressources Aquatiques Continentales, 50, avenue de Verdun - 33612 CESTAS Cedex, France.

E-mail : Christine.gazeau@ cemagref.fr

(2) Adresse actuelle : Institut National de la Recherche Agronomique, UMR Ecobiologie et Qualité des Hydrosystèmes Continentaux, 65 rue de St Brieuc, 35042 RENNES Cedex, France.

\section{RÉSUMÉ}

Pour éviter les risques de dérives méthodologiques ou d'hétérogénéité au fil du temps ou des opérateurs, il est nécessaire pour une bonne qualité des travaux réalisés en écologie comme en dynamique des populations de disposer de protocoles d'acquisition de données formalisés suffisamment détaillés.

Dans le cadre de l'analyse du fonctionnement de la population de grande alose Alosa alosa du bassin versant Gironde-Garonne-Dordogne (France), nous avons mené un travail de standardisation des différentes étapes de l'acquisition des données d'échantillonnage.

Après une analyse détaillée et exhaustive de la démarche d'acquisition, nous avons identifié trois parties : la procédure d'échantillonnage, le mode opératoire des prélèvements sur le terrain et le mode opératoire de la préparation et du montage des écailles au laboratoire. Chacune de ces parties est présentée chronologiquement, le cheminement de l'information est précisé au long de la chaîne des transcripteurs (poissons, écailles, données) et certaines étapes clés sont précisées à titre d'illustration.

Les contraintes supplémentaires engendrées par cette formalisation sont essentiellement dues au temps requis pour la rédaction des protocoles selon les normes de l'assurance qualité ; en revanche au cours de la réalisation de l'étude, cette démarche apporte un gain de temps et une amélioration de fiabilité des données en la sécurisant et en la standardisant. C'est également une étape indispensable pour envisager l'accréditation de laboratoire.

Mots-clés : démarche qualité, méthodologie, grande alose, échantillonnage. 


\title{
SET-UP OF «A QUALITY ASSURANCE » \\ AS PART OF SAMPLING OF AN ALLIS SHAD (ALOSA ALOSA) POPULATION.
}

\begin{abstract}
If the protocols of data acquisition are not formalized enough, in ecology or in population dynamics, we run the risk that, with time and different operators, there will be a non-measurable drift. The consequences may be serious when it comes to interpretations. To limit these risks, we have tried to standardize all stages of sampling data acquisition on functioning of the Alosa alosa population in the Gironde-Garonne-Dordogne drainage basin.
\end{abstract}

After a detailed analysis of the acquisition stage, we have split it into 3 main parts : the sampling procedure, the operative mode of sampling in the field and the operative mode of cleaning and mounting scales in the laboratory.

Each part is shown chronologically ; each task is explained and the exact path of information is specified throughout the transcriber chain (fish, scales, and data).

Supplementary difficulties are essentially due to the time necessary to write the protocol in due form. On the other hand, at the moment of the carrying out the study, security and standardization save time for the data reliability.

Key-words : quality stage, methodology, allis shad, sampling.

\section{INTRODUCTION}

L'unité Ressources Aquatiques Continentales du Cemagref mène des recherches sur l'écologie et la dynamique de population de la grande alose (Alosa alosa) du bassin versant Gironde-Garonne-Dordogne depuis plusieurs années (TAVERNY, 1991 ; ROCHARD, 1992 ; MARTIN-VANDEMBULCKE, 1999). Les données recueillies (longueur, sexe, âge, etc.) grâce aux campagnes d'échantillonnage des géniteurs lors des saisons de migrations 1994-1996 ont permis l'élaboration d'un modèle de dynamique de population (MARTIN-VANDEMBULCKE, 1999). Afin de disposer des données nécessaires à la validation de ce modèle, 4 campagnes annuelles d'échantillonnages complémentaires ont été programmées (1997-2000). A cette occasion nous avons souhaité ébaucher les bases de l'application d'une démarche qualité à l'acquisition de ces données. L'objectif de cette démarche est d'assurer la fiabilité, la traçabilité et la répétitivité sans dérive des interventions, de façon à aboutir à une meilleure qualité des données et donc potentiellement à une meilleure qualité des résultats. Ce document illustre une première formalisation des étapes constituant la chaîne d'acquisition des paramètres biométriques et démographiques et de leur organisation; depuis la planification des campagnes jusqu'au stockage des échantillons et au transfert des données aux biologistes chargés de les analyser. Une fois validée par le responsable du projet, l'application de chaque partie s'impose aux différents acteurs techniques. Cette note n'a pas vocation à présenter de façon détaillée l'ensemble des méthodes impliquées dans la réalisation de cet échantillonnage (les aspects statistiques de l'échantillonnage notamment ne sont pas présentés), mais vise à illustrer et à discuter l'application d'une démarche qualité dans le cadre de projet contenant un grand nombre de tâches de nature très différente. 


\section{MATÉRIEL ET MÉTHODES}

Le fond du protocole d'échantillonnage (nombre de spécimens, sex-ratio, période, lieux, etc.) est issu de travaux préalablement menés sur les populations d'aloses (MENNESSON-BOISNEAU, 1990 ; TAVERNY, 1991 ; MARTIN VANDEMBULCKE, 1999 ; LE CORRE, 1999 ; LAMBERT et al., 2001).

Les canevas standards établis pour la rédaction des documents qualité au Cemagref (ANONYME, 1998) ont été appliqués au protocole d'échantillonnage de la grande alose et ont guidé l'organisation du travail. Afin d'illustrer la démarche, nous avons choisi de présenter l'acquisition des données en utilisant des organigrammes correspondant à une procédure d'échantillonnage, à un mode opératoire pour les prélèvements sur le terrain et à un mode opératoire pour la préparation et le montage des écailles au laboratoire. Dans ces organigrammes seulement certaines parties ont été détaillées, afin de montrer le degré de précision requis. En pratique l'ensemble de la démarche doit être défini à ce niveau de précision. Au travers de ces organigrammes, on visualise nettement de quelle façon les données changent de nature et prennent de la valeur au fur et à mesure de leur cheminement dans une "chaîne de transcripteurs " (LATOUR et WOOLGAR, 1996).

On définit une procédure comme l'ensemble des règles et des modalités d'action qui doivent être respectées pour obtenir un résultat déterminé. Ses règles et ses modalités y sont consignées. Un mode opératoire est un document qui décrit la manière de réaliser une ou des opérations techniques.

\section{RÉSULTATS}

La chaîne d'acquisition peut se résumer en trois organigrammes séquentiels (Figure 1 ; Figure 2 ; Figure 3) correspondant chacun à une partie de la démarche.

La procédure d'échantillonnage (Figure 1) décrit la méthode d'organisation pour le déroulement de l'étude. Cette première étape précise explicitement les zones d'échantillonnage (Estuaire, Garonne et Dordogne), la période d'échantillonnage (pendant toute la migration, avec un effort plus important en début et en fin de migration), ainsi que la fréquence d'échantillonnage (une fois par semaine sur chaque site). Ces paramètres ayant été définis ou calculés par ailleurs. Elle précise également la démarche retenue pour organiser une sortie d'échantillonnage : du contact téléphonique avec le pêcheur, jusqu'au rendez-vous sur le site de pêche. Cette partie de la chaîne de transcripteurs permet de passer de «la personne qui conçoit l'étude » au «carnet de rendez-vous " où sont consignés les contacts pris par le technicien, les réponses des pêcheurs, les dates et heures de rendez-vous, etc. 


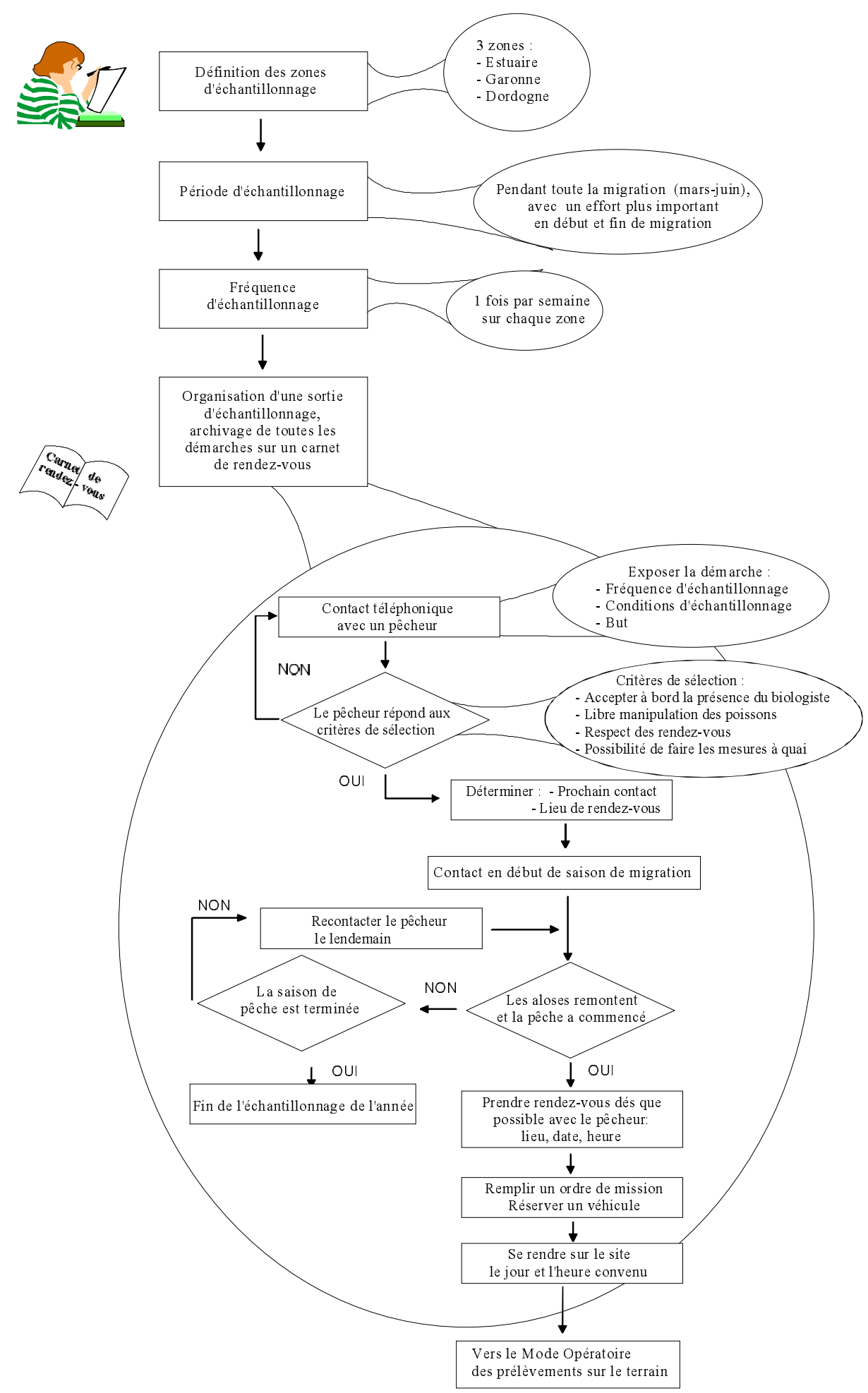

\section{Figure 1}

Procédure de préparation de l'échantillonnage auprès des pêcheurs.

\section{Figure 1}

Procedure to prepare the field sampling with fishermen. 
Le mode opératoire des prélèvements sur le terrain (Figure 2) contient l'inventaire du matériel nécessaire (non détaillé ici), les indications utiles pour le choix et le nombre de poissons à échantillonner. A partir de travaux de références, il précise la méthode pour effectuer les relevés méristiques (TAVERNY, 1991 ; LE CORRE, 1999), la détermination du sexe, les prélèvements des écailles (BAGLINIERE et al., 2001) et le relevé des informations sur le terrain. II donne également les instructions pour la saisie ultérieure au laboratoire des informations dans les supports informatiques avec des formats adéquats et standardisés et pour le stockage des échantillons d'écailles. Cette partie de la chaîne de transcripteurs permet de passer de «l'alose capturée » au « sachet-écaille » et au fichier informatique contenant le « Carnet de pêche ».

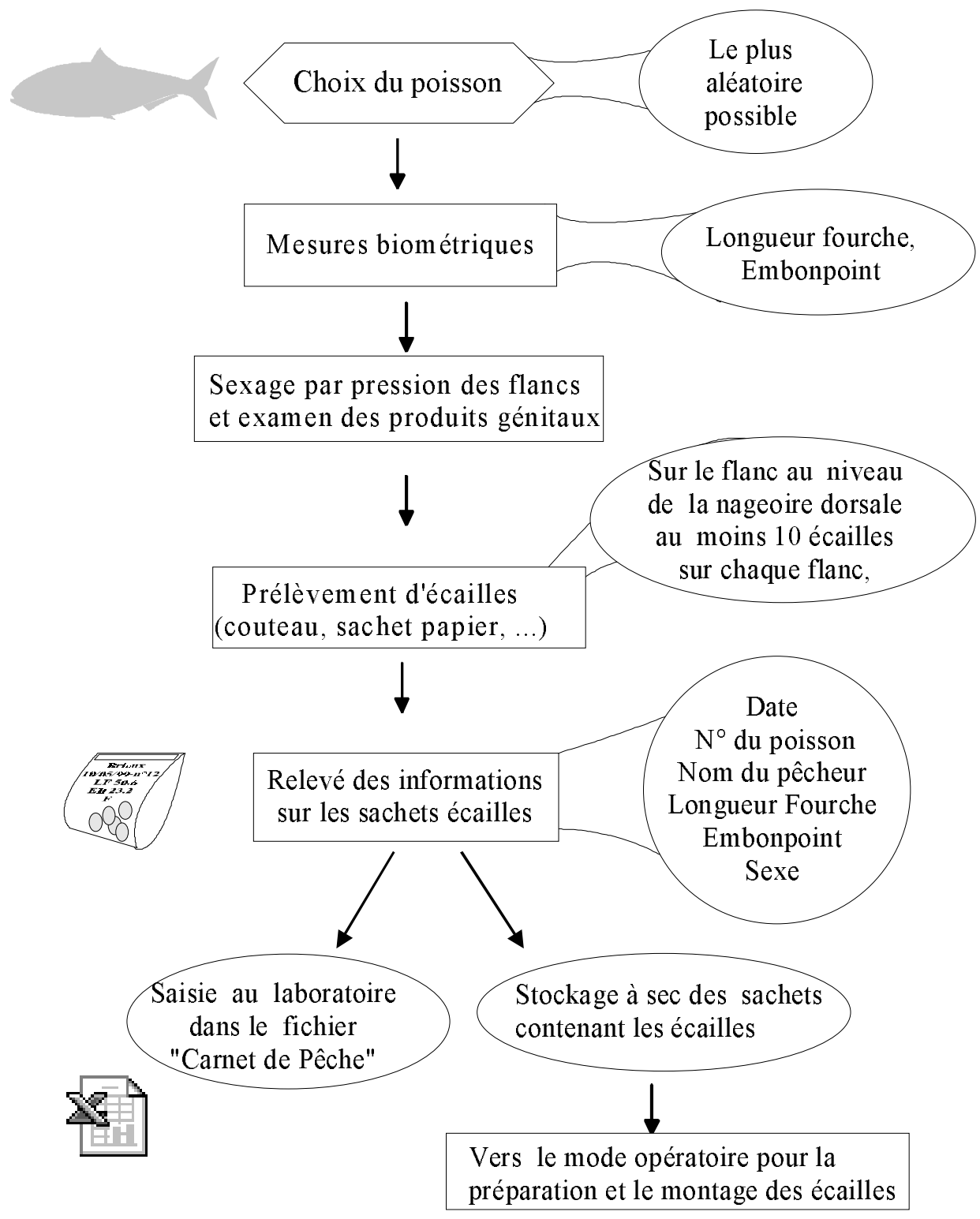

\section{Figure 2}

Mode opératoire concernant les mesures et prélèvements effectués sur le terrain.

\section{Figure 2}

Operative mode of measuring and field sampling. 
Le mode opératoire pour la préparation et le montage des écailles (Figure 3) précise le traitement des écailles au laboratoire. Il dresse l'inventaire du matériel nécessaire et il décrit les aspects techniques de la méthode retenue pour la préparation et le montage des écailles (BAGLINIERE et al., 2001). II renseigne également sur le conditionnement et le stockage des lames supportant les écailles. Cette partie de la chaîne de transcripteurs permet de passer du « sachet-écailles » aux «lames » et au fichier informatique contenant les « mesures aloses».

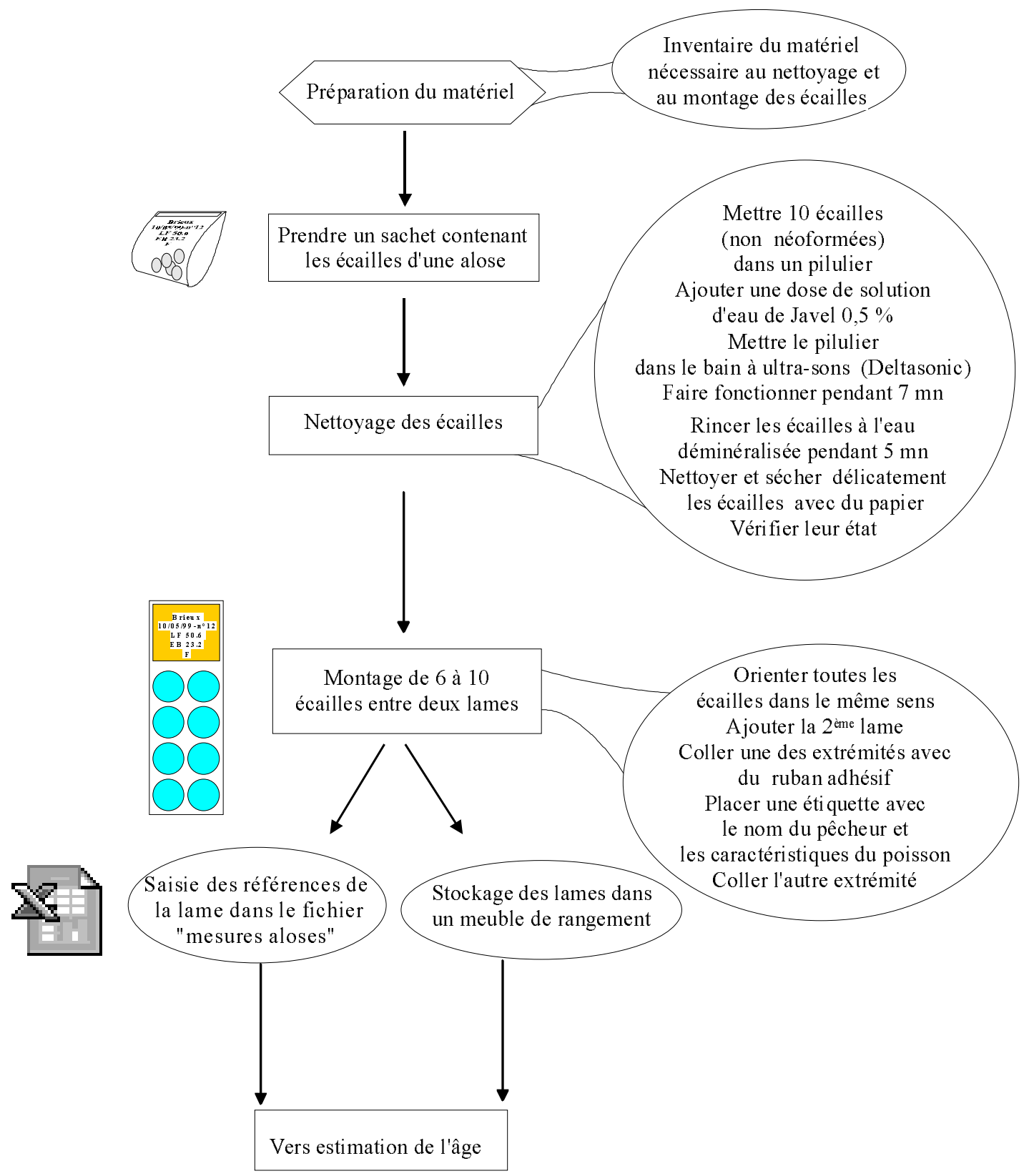

\section{Figure 3}

Mode opératoire pour la préparation et le montage des écailles au laboratoire.

\section{Figure 3}

Operative mode of scales preparation and mounting in laboratory. 
Sur la période (1997-2000), 30 sorties de terrain ont été réalisées en moyenne chaque année et ont permis l'obtention d'information concernant 600 aloses. La préparation, l'organisation et les sorties sur le terrain nécessitent 33 jours par an; le traitement au laboratoire, la saisie et l'archivage des données 30 jours par an.

Seulement cinq erreurs ont été relevées. II s'agissait dans tous les cas de l'oubli de l'inscription du sexe du poisson sur le « sachet-écailles ». Ceci correspond à moins de 1\% des aloses échantillonnées.

\section{DISCUSSION}

Au niveau de l'organisation et de la synchronisation du projet, les organigrammes nous ont permis de préciser le rôle de chacun dans la réalisation, de rendre autonome chaque partie dans son fonctionnement interne et de standardiser la réalisation du travail d'une année sur l'autre. On dispose ainsi d'une meilleure estimation du temps requis pour les différentes tâches, ce qui facilite la prévision et l'organisation du travail.

L'utilisation des organigrammes et la standardisation de l'archivage ont sécurisé la prise de données, ont accru la traçabilité « aptitude à retrouver l'historique, l'utilisation ou la localisation d'une entité au moyen d'identifications enregistrées ». Ceci permet au commanditaire et à la hiérarchie de contrôler les travaux.

Tout cela, conduit à la fiabilisation de la donnée. Cette fiabilisation n'est pas quantifiable pour le moment, mais pourra l'être sur le long terme.

L'apprentissage de la «méthode qualité », incluant l'intégration des notions de procédures, de modes opératoires, d'organigrammes, a été estimé à 10 jours. La rédaction des « documents qualités » relatifs à l'échantillonnage d'une population de grande alose (Alosa alosa) a nécessité 20 jours. Au moment d'une première mise en place d'une telle démarche, la contrainte de temps qu'impose la rédaction des protocoles standards est lourde mais elle se transforme peu à peu en gain de temps, de sécurité et de qualité dans les possibilités d'interprétation. La méthode sera par la suite applicable à d'autres travaux de recherche.

La chaîne de transcripteurs que nous avons présentée se poursuit logiquement par l'estimation de l'âge des aloses. Les difficultés de stockage et d'archivage des lames nous ont conduit à envisager l'utilisation de supports numériques qui fiabilisent la traçabilité, l'archivage et facilitent les lectures multiples.

\section{CONCLUSION}

Au fil du protocole, l'enrichissement progressif de l'information est fiabilisé par la démarche qualité. Nous avons désormais un protocole d'échantillonnage standardisé, permettant l'acquisition de données sur le long terme. La mise en place d'une telle démarche, en limitant les risques de dérive, nous permet de nous préparer à une éventuelle accréditation de notre laboratoire. Ceci nous conduit à envisager l'extension de ce type de formalisation pour d'autres projets. 


\section{REMERCIEMENTS}

Nous tenons à remercier Eric BRIEUX, Philippe et Sébastien GAUTIER et Christophe HUGUET, pêcheurs professionnels, pour leur collaboration et leur accueil fort sympathique ; sans eux ces échantillonnages n'auraient pas été possibles.

Nous remercions également Serge FISHER et Patrick LAMBERT du Cemagref pour leur précieuse collaboration. Le premier pour ses judicieux conseils dans le domaine de la qualité ; le second pour ses relectures constructives.

\section{BIBLIOGRAPHIE}

ANONYME, 1998. Manuel Qualité du Groupement de Bordeaux, $75 \mathrm{p}$.

BAGLINIĖRE J.L., SABATIÉ M.R., APRAHAMIAN M.W., ALEXANDRINO P., APRAHAMIAN C.D., ASSIS C.A., CASSOU-LEINS J.J., LE CORRE M., MENNESSON-BOISNEAU C., MARTIN-VANDEMBULCKE D., ROCHARD E., TEIXERA C., 2001. Guide pour l'interprétation des écailles et l'estimation de l'âge chez les aloses (Alosa spp.) de la façade Atlantique-Est et de la MéditerranéeOuest. Bull. Fr. Pêche Piscic., 357/360, 485-531.

LAMBERT P., MARTIN VANDEMBULCKE D., ROCHARD E., BELLARIVA J.L., CASTELNAUD G., 2001. Age à la migration de reproduction des géniteurs de trois cohortes de grandes aloses (Alosa alosa) dans le bassin versant de la Garonne (France), Bull. Fr. Pêche Piscic., 362/363, 973-987.

LATOUR B., WOOLGAR S., 1996. La vie de laboratoire - la production des faits scientifique. ed. La Découverte/Poche, $300 \mathrm{p}$.

LAVARDE P., 1999. Une politique pour la qualité au Cemagref. In: Manuel Qualité du Cemagref.

LE CORRE M., 1999. Eco-biologie et génétique des populations d'aloses (Alosa fallax spp.) de la Méditerranée française. Thèse de doctorat de l'Ecole Nationale Supérieure Agronomique de Rennes. $189 \mathrm{p}$.

MARTIN-VANDEMBULCKE D., 1999. Dynamique de la population de la grande alose (Alosa alosa L. 1758) dans le bassin versant Gironde-Garonne-Dordogne (France) : analyse et prévision par modélisation. Thèse de doctorat, Université de Toulouse, $115 \mathrm{p}$.

MENNESSON-BOISNEAU C., 1990. Migration, répartition, reproduction et caractéristiques biologiques des aloses Alosa sp. dans le bassin de la Loire. Thèse de doctorat, Université de Rennes I, $106 \mathrm{p}$.

ROCHARD E., 1992. Mise au point d'une méthode de suivi de l'abondance des amphihalins dans le système fluvio-estuarien de la Gironde, application à l'étude écobiologique de l'esturgeon européen Acipenser sturio. Thèse de doctorat, Université de Rennes I, $315 \mathrm{p}$.

TAVERNY C., 1991. Contribution à la connaissance de la dynamique des populations d'aloses (Alosa alosa et Alosa fallax) dans le système fluvio-estuarien de la Gironde: pêche, biologie et écologie. Etude particulière de la dévalaison et de l'impact des activités humaines. Thèse de doctorat, Université de Bordeaux I, $451 \mathrm{p}$. 